\title{
Relação peso-comprimento de Orthopristis ruber (Cuvier) (Teleostei, Haemulidae) na Baia de Sepetiba, Rio de Janeiro, Brasil
}

\author{
André L. B. dos Santos ${ }^{1}$, André L. M. Pessanha ${ }^{1}$, Marcus R. da Costa ${ }^{1}$ \& Francisco G. Araújo ${ }^{1,2}$ \\ ${ }^{1}$ Laboratório de Ecologia de Peixes, Universidade Federal Rural do Rio de Janeiro. Antiga Rodovia Rio-São Paulo, km 47, \\ 23851-970 Seropédica, Rio de Janeiro, Brasil. \\ ${ }^{2}$ Autor para correspondência. E-mail: gerson@ufrri.br
}

\begin{abstract}
Length-weight relationship of Orthopristis ruber (Cuvier) (Teleostei, Haemulidae) in the Sepetiba Bay, Rio de Janeiro, Brazil. The lenght-weight relationship of Orthopristis rubber $(C u v i e r, 1830)$ in the Sepetiba Bay $\left(22^{\circ} 54^{\prime}-23^{\circ} 04^{\prime} \mathrm{S} ; 43^{\circ} 34^{\prime}-44^{\circ} 10^{\prime} \mathrm{W}\right)$, Rio de Janeiro was determined. It aims to contribute to morphometrics knowledge of this species in a semi-closed coastal area and to supply basis for comparisons with others environment. Fishes were collected by beach seine (juveniles) and otter trawl (adults) from October 1998 to September 1999. The equation found was $\mathrm{W}=0,000006 \mathrm{~L}^{3,1368}$ for males and $\mathrm{W}=0,000006 \mathrm{~L}^{3,1403}$ for females. Positive allometry was found for both sexes and sex rate was 1:1. The comparatively high values for allometric coefficient indicate high investment in growth, which can be due to relatively small size of the examined fish populations, or a mechanism to overcome stress conditions in the area.
\end{abstract}

KEY WORDS. Grunts, allometry, coastal fishes, bays, morphometrics.

RESUMO. A relação peso-comprimento de Orthopristis ruber (Cuvier, 1830) na Baia de Sepetiba $\left(22^{\circ} 54^{\prime}-23^{\circ} 04^{\prime}\right.$ 'S; $43^{\circ} 34^{\prime}-44^{\circ} 10^{\prime} \mathrm{W}$ ), Rio de Janeiro foi determinada. Este trabalho objetiva contribuir com o conhecimento sobre a morfometria desta espécie na baía e fornecer bases para comparações com outros ambientes. Os peixes foram coletados em programas de amostragens de arrasto de praia (juvenis) e arrasto de fundo (adultos) entre outubro de 1998 e setembro de 1999. A equação encontrada foi $W=0,000006 L^{3,1368}$ para machos e $W=0,000006 L^{3,1403}$ para fêmeas. Alometria positiva foi encontrada para machos e fêmeas e a proporção sexual foi de 1:1. Os altos valores para o coeficiente alométrico indicam alto investimento no crescimento, que pode ser devido ao pequeno tamanho relativo dos espécimes da população analisada, ou ser um mecanismo para suportar as condições de estresse nesta área.

PALAVRAS CHAVE. Cocoroca, alometria, peixes costeiros, baías, morfometria.

Orthopristis ruber (Cuvier, 1830), vulgarmente conhecido como cocoroca, é o mais abundante representante da família Haemulidae na Baía de Sepetiba $\left(22^{\circ} 54^{\prime}-23^{\circ} 04^{\prime}\right.$ 's; $43^{\circ} 34^{\prime}$ $\left.44^{\circ} 10^{\prime} \mathrm{W}\right)$. Esta baía constitui uma área semi-fechada na costa do Estado do Rio de Janeiro, que serve de criação para várias espécies de peixes costeiros (ARAújo et al. 1998). Seu habitat é bastante diverso, principalmente em áreas de fundos de pedras, praias arenosas, baias e regiões estuarinas, distribuindose desde o Caribe até ao sul do Brasil (Menezes \& Figueiredo 1980).

A relação peso-comprimento é um importante parâmetro das populações de peixes, e suas aplicações variam desde a estimativa do peso de um indivíduo, conhecido seu comprimento, até indicações da condição dos peixes, além de ser útil como indicadora do acúmulo de gordura, desenvolvimento das gônadas (Le CRen 1951). Também serve servindo para indicar investimentos energéticos para crescimento e ou reprodução, fenômenos normalmente cíclicos nas populações de peixes. Comparações morfométricas interespecíficas e interpopulacionais das espécies de peixes podem ser derivadas do coeficiente de alometria que indica eventuais mudanças na forma ao longo do desenvolvimento ontogenético. Adicionalmente, esta relação pode servir de base para comparar o grau de estresse ou diferentes condições ambientais entre peixes de ampla distribuição geográfica (BOLGER \& CONNOLLY 1989). Os coeficientes "a" e "b" da relação peso-comprimento podem diferir, não somente entre as espécie de peixes mas também entre estoques da mesma espécie (BAgenal \& Tesch 1978 apud NARAHARa et al. 1985).

O objetivo deste presente trabalho foi determinar a relação peso-comprimento para a população de O. ruber da Baia de Sepetiba, visando contribuir com o conhecimento da morfometria desta espécie, bem como estabelecer bases para comparações com outras populações da costa brasileira.

Revista Brasileira de Zoologia 21 (2): 185-187, junho 2004 

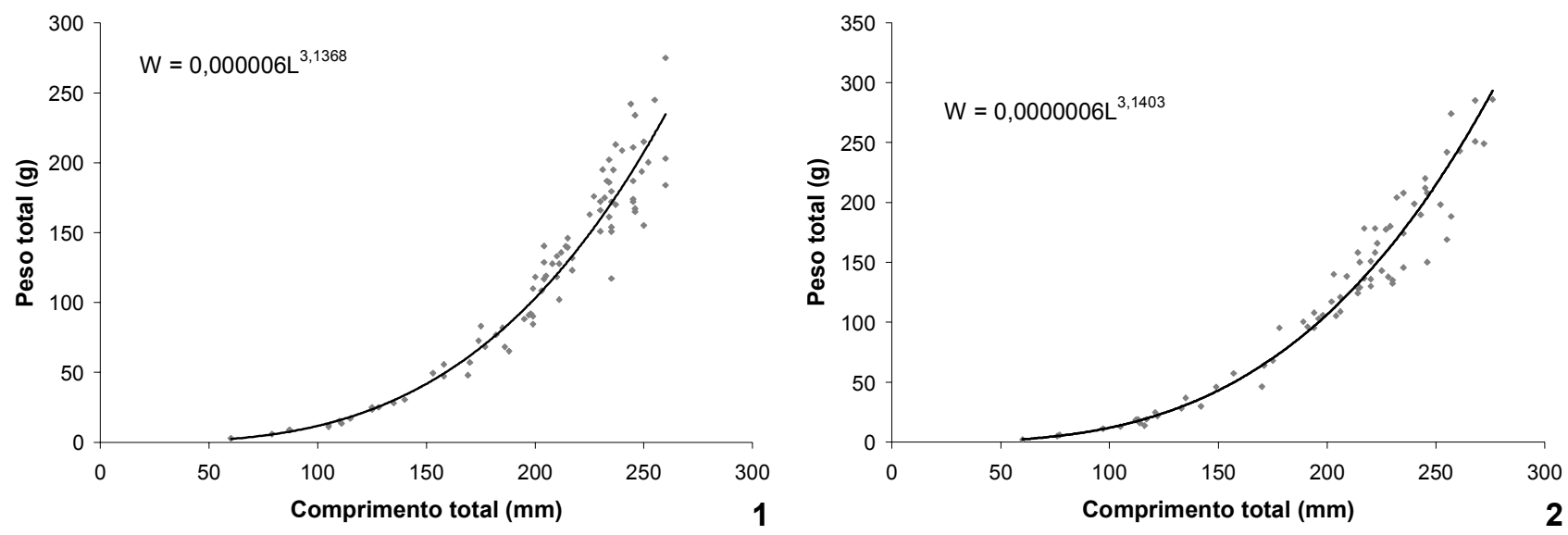

Figuras 1-2. Relação peso-comprimento de (1) machos e (2) fêmeas de Orthopristis ruber na Baía de Sepetiba, 1998/1999.

\section{MATERIAL E MÉTODOS}

\section{Área de estudos}

A Baía de Sepetiba está localiza-se no Estado do Rio de Janeiro $\left(22^{\circ} 54^{\prime}-23^{\circ} 04^{\prime} \mathrm{S} ; 43^{\circ} 34^{\prime}-44^{\circ} 10^{\prime} \mathrm{W}\right)$, com área de aproximadamente $450 \mathrm{~km}^{2}$. Apresenta forma alongada, limitando-se a Norte e a Leste pelo continente, ao Sul pela Restinga de Marambaia e a Oeste, pela Baía de Ilha Grande. Seu maior comprimento é de $43 \mathrm{~km}$ no sentido leste-oeste e sua maior largura é de $17 \mathrm{~km}$ no sentido norte-sul, com perímetro de aproximadamente $123 \mathrm{~km}$. Atualmente a Baía de Sepetiba encontra-se sob forte pressão antrópica, devido à expansão industrial, turística e aumento dos contingentes populacionais em suas imediações, com a decorrente alteração ambiental causadas por efluentes urbano-industrial de fontes variadas e difusas, bem como pela alteração da paisagem. Isto reflete diretamente na qualidade ambiental, com influência direta na produtividade pesqueira e na qualidade de vida na área.

\section{Análise de dados}

Foram utilizados 156 exemplares, sendo 84 machos e 72 fêmeas, provenientes de um programa de amostragens de arrastos de fundo realizados na Baia de Sepetiba, no período de outubro de 1998 a setembro de 1999. Os arrasto de fundo foram realizados com barco tipo arrasteiro de $12 \mathrm{~m}$ de comprimento com três amostragens aleatórias em cada zona da baia (interna, central e externa). Os peixes foram fixados em formol a $10 \%$ e, após 48 horas, transferidos para álcool a 70\%. A identificação foi de acordo com Menezes \& Figueiredo (1980). Todos os indivíduos foram medidos ao comprimento total ( $\mathrm{mm}$ ) e pesados ( $\mathrm{g}$ ). Os machos e fêmeas foram separados através da análise das gônadas, identificadas sob o estereomicroscópico. Os dados foram logaritmizados, e as equações das retas foram determinadas pelo método dos mínimos quadrados. Para a relação pesocomprimento foi aplicado a formula: $\mathrm{W}=\varnothing \mathrm{L}^{\theta}$ onde, $\varnothing=10^{\mathrm{a}} ; \theta$ = $\mathrm{b}, \mathrm{W}$ é o peso total, e L, o comprimento total (SANTOS 1978).

\section{RESULTADO E DISCUSSÃO}

O comprimento total dos peixes examinados variou de 60 a $276 \mathrm{~mm}$ para fêmeas, e de 60 a $260 \mathrm{~mm}$ para machos. O peso total variou de 2,21 a $286 \mathrm{~g}$ para fêmeas e 2,89 a $275 \mathrm{~g}$ para machos. A proporção sexual foi 1:1. A relação peso-comprimento foi: $\mathrm{W}=0,000006 \mathrm{~L}^{3,1368}$ para machos (Fig. 1); e $\mathrm{W}=$ $0,000006 \mathrm{~L}^{3,1403}$ para fêmeas (Fig. 2).

O coeficiente de alometria é um indicador da velocidade de inflexão da curva para atingir os valores assintóticos, isto é, quando o crescimento em comprimento passa a apresentar um incremento irrelevante em relação ao peso (Le cren 1951). Comparando-se o coeficiente de alometria dos machos $(\theta=3,1368)$ e das fêmeas $(\theta=3,1403)$ com o valor de referência para isometria (3) verificou-se que ambos os sexos diferiram significantemente de 3 (alometria positiva). Os valores encontrados para a Baía de Sepetiba foram comparativamente maiores do que aqueles encontrados para a população do estuário da Laguna dos Patos, RS $(\theta=2,6859)$ determinado por (HAIMOVICI $\&$ Velasco 2000), os quais não apresentaram valores para os sexos em separado, porém o baixo coeficiente de alometria sugere alometria negativa. Uma das explicações para tais diferenças pode ser atribuída a estrutura de tamanho das duas populações. Enquanto a estrutura de tamanho dos peixes da Baía de Sepetiba variou de 60 a $276 \mathrm{~mm}$, no estuário da Laguna dos Patos, um ambiente relativamente melhor preservado, os indivíduos variaram de 145 a $275 \mathrm{~mm}$ de comprimento total. Populações de peixes de maior estrutura de tamanho, normalmente apresentam menor coeficiente de alometria quando comparadas com populações de menor estrutura de tamanho, uma vez que estes últimos ainda estão alocando energia para crescer. A estratégia de um mais rápido crescimento, visando atingir a maturidade sexual com menores tamanhos, também poderia estar sendo desenvolvida por $O$. ruber na Baía de Sepetiba, visando manter as populações face ao crescente estado de estresse que a Baía vem sofrendo nos últimos anos devi- 
do às influências antrópicas. Atualmente, mais de 100 indústrias dos mais variados tipos, de médio e grande porte, descargam grandes quantidades de substâncias potencialmente tóxicas na baia, inclusive metais pesados (SEMA 1998), o que provavelmente constitui uma considerável pressão sobre os peixes deste ambiente.

\section{AGRADECIMENTOS}

Agradecemos a Márcia Cristina Costa de Azevedo, Antonio Gomes da Cruz-Silva, Pablo Mendonça, Francisco José Costa Guimarães, Rosana Rodrigues Milagre, Hamilton Hissa Pereira, Leonardo Mitrano Neves, Iracema David Gomes, André Vasconcellos Araújo e Márcio de Araújo Silva pelas ajudas nas coletas de campo. O Conselho Nacional de Desenvolvimento Científico e Tecnológico-CNPq e a Fundação Carlos Chagas de Amparo à Pesquisa do Estado do Rio de Janeiro - FAPERJ financiam parcialmente o Projeto Bioecologia dos Peixes da Baía de Sepetiba, do qual este trabalho faz parte.

\section{REFERÊNCIAS BIBLIOGRÁFICAS}

Araújo, F.G.; A.G. Cruz-filho; M.C.C. Azevedo \& A.C.A. Santos. 1998. Estrutura da comunidade de peixes demersais da Baia de Sepetiba, RJ. Revista Brasileira de Biologia, São Carlos,
58 (3): 417-430.

Bolger, T. \& P.L. Connolly. 1989. The selection of suitable indices for the measurement and analysis of fish condition. Journal Fish Biology, Dunscore, 34: 171-182.

Haimovici, M \& G. Velasco. 2000. Length-weight relationship of marine fishes from Southern Brazil. Naga, The Iclarm Quarterly, Makati City, 23 (1): 19-23.

LE CREN, E.D. 1951. The lenght-weight relationship and seasonal cycle in gonad and conditions in the perch Perca fluviatilis. Journal Animal Ecology, Heslington, 20 (2): 201.

Menezes, N.A \& J.L. Figueiredo. 1980. Manual de peixes marinhos do sudeste do Brasil. IV Teleostei (3). São Paulo, Museu de Zoologia, Universidade São Paulo, 96p.

Nahara, M.Y.; H.M. Godinho; N. Fenerich-verani \& E. Romagosa. 1985. Relação Peso-comprimento e fator de condição de Rhamdia hilarii (Valenciennes, 1840) (Osteichthyes, Siluriformes, Pimelodidae). Boletim Instituto Pesca, São Paulo, 12 (4): $13-22$.

SANTOS, E.P. 1978. Dinâmica de população aplicada à pesca e à piscicultura. São Paulo, Hucitec, Universidade de São Paulo, 129p.

SEMA. 1998. Macroplano de gestão e Saneamento Ambiental da bacia da Baia de Sepetiba. Rio de Janeiro, Secretaria de Estado de Meio Ambiente do Rio de Janeiro, vol. 1.

Recebido em 04.IV.2003; aceito em 26.IV.2004. 